\title{
Dynamic Behavior Selection Model based on Emotional States for Conbe-I robot
}

\author{
Wisanu Jitviriya and Jiraphan Inthiam \\ Computer Science and Systems Engineering, Kyushu Institute of Technology. \\ 680-4, Kawazu, Iizuka, Fukuoka, 820-8502, Japan. \\ wisanu@mmcs.mse.kyutech.ac.jp,jiraphan@mmcs.mse.kyutech.ac.jp \\ Eiji Hayashi \\ Mechanical Information Science and Technology, Kyushu Institute of Technology. \\ 680-4, Kawazu, Iizuka, Fukuoka, 820-8502, Japan. \\ haya@mse.kyutech.ac.jp
}

\begin{abstract}
Currently, the rapid development of non-industrial robots that are designed with artificial intelligence (AI) methods to improve the robotics system is to have them imitate human thinking and behavior. Therefore, our works have focused on studying and investigating the application of brain-inspired technology for developing the conscious behavior robot (Conbe-I). We created the hierarchical structure model, which is called "Consciousness-Based Architecture: CBA" module, but it has limitation in managing and selecting the behavior that only depends on the increase and decrease of the motivation levels. Consequently, in this paper, we would like to introduce the dynamic behavior selection model based on emotional states, which develops by Self-organizing map learning and Markov model in order to define the relationship between the behavioral selection and emotional expression model. We confirm the effectiveness of the proposed system with the experimental results.
\end{abstract}

Keywords: Behavior selection model, Self-organizing map (SOM) learning, Markovian model.

\section{Introduction}

Nowadays, the focus of research of service robots is a development of the robots that are able to express emotions, imitate human thinking and change their behavior based on variety surrounding environment. However, development of these robots that are still a huge challenge when they have interaction with humans. Therefore, Hayashi laboratory's works [1]-[2] have focused on studying and investigating the application of brain-inspired technology by developing the robots with a hierarchical structure module based on Tran Duc Thoa's research [3]. This model is called "Consciousness-Bead Architecture (CBA)" model which relates between consciousness field and behavior group. But the conventional (CBA) model has limitation in managing and selecting the behavior that only depends on the increase and decrease of the motivation levels. For a truly effective of the robotic system, the robots should not only perform the behavior, but also express the appropriate emotional state. Thus, the general objective of this research, we would like to introduce the proposed system, which can autonomously select the suitable behavior based on transition of emotional states.

Our paper is organized as follows: Chapter 2 explains the autonomous behavior selection system which has three processes. In Chapter 3 , our results are shown how our robot can choose behavior depending on 
its emotional states. In the final section we conclude the paper and describe our future works.

\section{Autonomous Behavior Selection System}

In this paper, we would like to propose the behavior selection model which autonomously selects and performs the suitable action based on variation of emotional expression. The proposed system has three major processes as described below.

\subsection{Computation dopamine and robot's motivation}

\subsubsection{Determination of occurring dopamine model}

The Conbe-I robot uses only the acquired images from the CCD camera and web camera for operating the actions and emotional expression in recognition process. The robot is not usable the other sensors (tactile sensor or laser range finder sensor). Thus, the robot can evaluate the rough position of the target object by only using the image processing. The system may simplify the acquired images by dividing into four color groups: green, blue, flesh-color and the other colors. With the simplification, the sequential recognition process has the reduced image size, the changed color model of image from RGB method to HSV method, the labeling process based on the color of visual information and the calculation of the position of the target object. Then, the visual information (the color, shape, size, labeling and distance of the target object) is used to calculate and form the naturally occurring dopamine waveform [4]. The computation of the typical pattern of dopamine model is explained. The dopamine waveform can be evaluated and divided into rise and fall portions. The rising part and falling part are created by the $2^{\text {nd }}$ order and $1^{\text {st }}$ system equations as shown in Eq. (1) and Eq. (2) respectively, where the input variable $x(t)$ is an accelerator of dopamine, the output variable $y(t)$ is a naturally dopamine, $\zeta$ is damping factor, $\omega_{n}$ is the natural frequency and $T_{c}$ is time constant.

$$
\begin{gathered}
y^{\prime \prime}(t)+2 \zeta \omega_{n} y^{\prime}(t)+\omega_{n}^{2} y(t)=\omega_{n}^{2} x(t) \\
T_{c} y^{\prime}(t)+y(t)=x(t)
\end{gathered}
$$

\subsubsection{Calculation of the robot's motivation}

Since the computation of the naturally occurring dopamine as described above, the sum of positive and negative dopamine values that is used as the input variable for calculating the robot's motivation. The motivation waveform can estimate by the $2^{\text {nd }}$ order system of differential equation as expressed by Eq. (3).

$$
\begin{aligned}
& \text { Motivation } "(t)+2 \zeta \omega_{n} \text { Motivation }^{\prime}(t) \\
& \qquad \omega_{n}^{2} \text { Motivation }(t)=\omega_{n}^{2} \text { Total_dopamine }(t)
\end{aligned}
$$

\subsection{Classification emotions and behaviors based on Self-organizing map (SOM) learning}

After recognition process, the robot's motivation and the visual information are used to define as the input parameters of the Self-Organizing Map (SOM) learning in order to generate the behavior and emotion maps. The robot is set the nine behaviors and six emotions. To classify and select the most appropriate behavior and emotion correspond to the surrounding environment. The results of the SOM learning the will be verified.

\subsubsection{SOM algorithm}

The Self-Organizing Map is a neural network architecture which is proposed by T. Kohonen [5]. It is well known as an effective pattern classifier that is an unsupervised learning method. The SOM method can provide topologically preserved mapping from highdimensional input data to a low - dimensional array based on competitive learning. The procedure of the basic SOM learning can explain by the following steps: Step 1:Each node must to be initialized with weight value based on setting the small standardized random value.

Step 2: Calculate and find the minimum Euclidean distance that is called "winner node or best matching unit (BMU)".

Step 3: After finding the BMU, the weight vectors of the BMU, and its topological neighbors are moved closer to the input vector. The new weight vectors are updated by Eq. (4), where $t$ is the training step index is, $\eta(t)$ is the learning rate, and $h_{j, c}(t)$ defines as the neighborhood kernel function around the winner nodes. 


$$
w_{j}(t+1)=w_{j}(t)+\eta(t) h_{j, c}(t)\left[\bar{x}-\bar{w}_{j}(t)\right]
$$

Step 4: The training step increases to $t+1$. Steps 2 to 4 are repeated with decreasing the width of the neighborhood function.

\subsubsection{Formation of behavior and emotion maps}

In order to verify the SOM learning, the motivation value, the slope of robot's motivation, the pixels of colors (green, blue and flesh colors) are defined as the input data for testing. For example, if the robot recognizes the green object, which is near the robot's hand. The testing data are the motivation flag $=1$ (positive level), the motivation value $=0.45$, the slope of robot's motivation $=0.003$, the motivation waveform is a positive slope $=1$, the green pixels $=0.6$, the blue pixels $=0.02$ and the flesh-color pixels $=0.2$. During the SOM learning process, we have not only considering the changing position of the winner nodes in behavior and emotion maps, but also observe the evolution of the form of both maps as shown in Fig.1 and Fig.2. The blue cells showed on behavior and emotion maps that were the winner nodes of each behavior and emotion pattern. The green cells represented as the response action according the testing input data. Therefore, in this situation, the robot should perform the action as "Approach", and express the emotion as "Happiness" at the same time. Because, the green object is a favorite object, thus it tries to possess the target object.

\subsection{Behavior selection based on Emotional States}

The last process is the behavioral-emotional selection process, which is modeled based on a Markov model [6]. The Markov model is memoryless property that means the next state will be changed based on a transition rule and depends only on the current state.

In this study, the Emotional Markovian model that consists of the six nodes representing the basic emotion states. The arrows are the probabilities of getting out of the states. The next emotional state can determine by Eq. (5), where, $A$ is the emotional state transition matrix.

$$
\text { Emotion }_{k+1}=A \cdot \text { Emotion }_{k}
$$

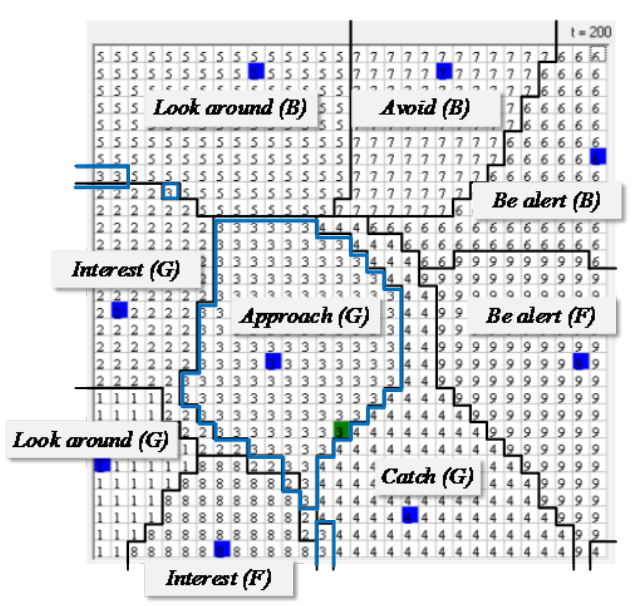

Fig.1 Behavior map

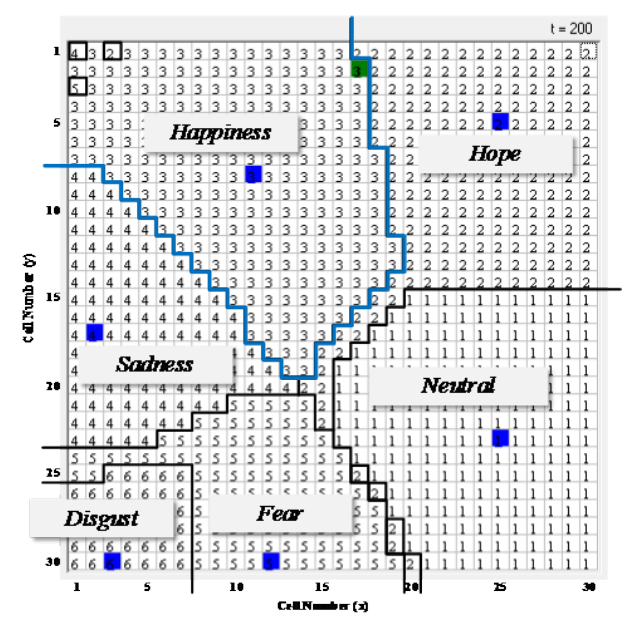

Fig.2 Emotion map

All elements of the emotional state transition matrix can be updated by the affective factors, which calculates by averaging the sum of the weights of individual group of behavior and emotional patterns in SOM learning process. In addition, the behavior selection module is based on its emotional expression, thus the behavior state will be selected by the emotional state that can estimate with Eq. (6), here, $C$ denotes as the behavioral state transition matrix.

$$
\text { Behavior }_{k+1}=C \cdot \text { Emotion }_{k+1}
$$

All element values in the behavioral state transition matrix ( $\left.c_{\text {behavior/emotion }}\right)$ can be modified later based on the behavioral and affective factors. 


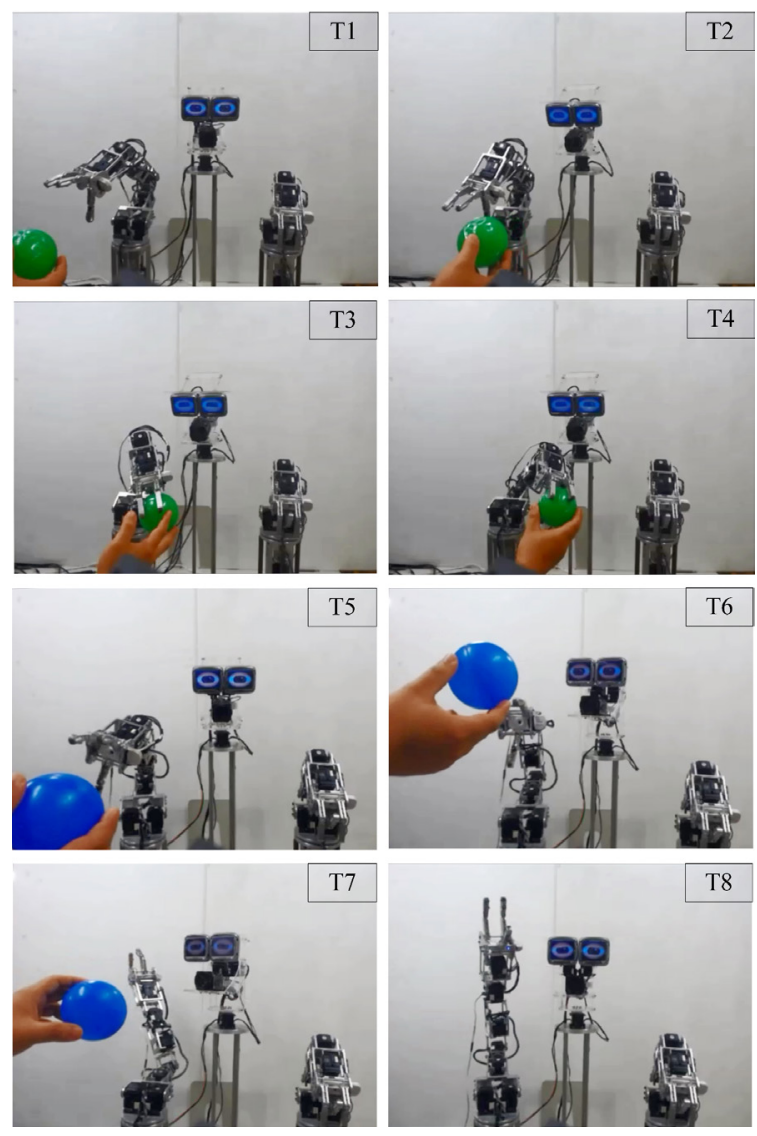

Fig.3 Behavioral and emotional selections at period T1-T8

\section{Experimental results}

The intelligent behavioral-emotional selection system was tested and evaluated, that confirmed its effectiveness in the surrounding environment.

The robot was verified its behavior and emotional expression when it recognized the favorite object (green object) and disliked object (blue object) in a realistic environment. Throughout the task period (T1-T8), the robot was able to act the appropriate behaviors and emotional expressions as illustrated in Fig.3. After the robot was started. The robot selected "Look around" and "Interest (Flesh)" actions when it recognized the flesh-color. At T1, the robot was able to capture the favorite object, thus the robot's motivation increased and the robot performed the behavior between "Look around" and "Interest", the robot also express emotion as "Neutral". From T2-T3, the robot performed the actions as "Interest" and "Approach" meanwhile it expressed "Hope" and "Happiness" emotions. Then, the blue object was presented in order to decrease the robot's motivation. At T5, the motivation model achieved in the negative level, during periods T5-T6, the robot felt unhappiness when it confronted the disliked object, the robot expressed "Sadness" and "Fear" emotions meanwhile it performed the action as "Look around" and "Alertness". After that (T6-T7), the blue object was moved closer to the robot, it felt dislike this situation, thus it performed "Avoidance" behavior and express "Disgust" emotion. Moreover, during period T6-T8, the robot expressed "Hope" emotion, when it did not see the disliked object or the robot's motivation increased to the positive level.

\section{Conclusions}

In this paper, the overview of the proposed system that consists of the recognition process, cognitive process and behavioral-emotional selection process. The system is executed by the Conbe-I robot in the realistic environment. All experimental results confirm the effectiveness of the proposed system such as the system can classify and generate the behavior and emotion maps based on the SOM learning method in the cognitive process, and the robot can suppress and change all emotional states based on the Markov model. In addition the robot can perform its behavior based on updating the emotional transition state. For the future work, we would like to combine the other neurotransmitters (noradrenaline and serotonin models) into the dopamine model in order to generate the dynamic emotional expression model.

\section{References}

1. N. Goto and E. Hayashi, "Design of Robotic Behavior that imitates animal consciousness," Journal of Artificial Life Robotics, vol. 12, pp. 97-101, 2008.

2. E. Hayashi, T. Yamasaki, and K. Kuroki, "Autonomous Behavior System Combing Motivation and Consciousness Using Dopamine," Proc. of $8^{\text {th }}$ Int. IEEE Symposium on Computational Intelligence in Robotics and Automation (CIRA 2009), pp. 126-131, 2009.

3. T. D. Thao, D. J. Herman, and D.V. Morano, Phenomenology and dialectical materialism (Boston Studies in the Philosophy of Science), D. Reidel Pub., 1986 
4. H. Kimura, "A trial to analyze the effect of an atypical antipsychotic medicine, risperidone on the release of dopamine in the central nervous system," J. of Aichi Medical University Association, vol. 33, no. 1, pp. 21-27, 2005.

5. T. Kohonen, "Self-organized formation of topologically correct feature map," J. Biological Cybernetics, vol. 43, pp. 56-69, 1982.

6. K. Koiji, and B. Martin, "Towards an emotion core based on a Hidden Markov Model," Proc. of $13^{\text {th }}$ IEEE International Workshop on Robot and Human Interactive Communication, Sep. 20-22, Kurashiki, Okayama, Japan, pp. 119-124, 2004. 